\title{
EL DEBATE DEL ABORTO Y SUS IMPLICACIONES EN LAS LEGISLACIONES MUNDIALES
}

Neus Sánchez Expósito

Institut Universitari d'Estudis de la Dona (IUED) neus.sanchez80@gmail.com

Recibido: 28-02-2010

Aceptado: 19-04-2010

\section{Resumen}

El aborto ha sido siempre un tema complejo que, en la actualidad, sigue suscitando intensos debates desde planteamientos muy dispares y posturas irreconciliables. Detrás de las diferentes líneas argumentativas para legitimar o no la interrupción voluntaria de la gestación existe un análisis de salud sexual y reproductiva desde una óptica moral con una concepción determinada de la "maternidad" que condiciona la jurisprudencia de los diferentes países. Muchas legislaciones permiten el aborto por cuestiones de salud, pero la defensa de la libertad de las mujeres lo reivindica como un derecho humano.

Palabras clave: aborto, libertad y autonomía, salud sexual y reproductiva, maternidad, dominación, legislación aborto.

\begin{abstract}
Abortion has been always a complex subject still carries on causing heated discussions from very disparate criteria and irreconcilable positions. Behind the different argumentative lines to legitimate, or not, the voluntary termination of pregnancy, there is an analysis on sexual and reproductive health from a moral viewpoint with a certain conception of "motherhood" that conditions all countries' jurisprudence. Many
\end{abstract}


legislations authorize abortion on heath matters, but the defense of the women's freedom demands it as a human right.

Key words: abortion, freedom and autonomy, sexual and reproductive health, motherhood, domination, abortion's legislation.

\section{Introducción}

En una cuestión tan compleja e introvertida como es el derecho a la interrupción voluntaria del embarazo, es necesario evitar simplificar el debate y mostrar los diversos argumentos que avalan los discursos fundamentales de los detractores y defensores de la legalización del aborto.

Inevitablemente, las mismas premisas de las diferentes posturas nos llevan a reflexionar sobre si existe un mismo nivel de debate que, en el caso de ser posible, nos permita conciliar posturas y llegar así a una legislación válida con la que se finalice las tensiones existentes en la sociedad alrededor de ésta controversia.

El objetivo de este trabajo es caracterizar, mediante sus argumentos, las posturas más emblemáticas que definen el debate sobre la legalidad o ilegalidad del aborto. Con ello, partiré a la reflexión sobre los diferentes niveles del debate, es decir, sobre la imposibilidad de conciliar los discursos debido a que no se habla, exactamente, de lo mismo: unos se centran en la mujer y otros en las "obligaciones" de la mujermadre y los derechos del no nacido.

Analizaré la construcción de la identidad de género alrededor de la maternidad, que condiciona las legislaciones de los diferentes países y que subyace en las diversas posturas con la intención de manifestar la necesidad no sólo de modificar las leyes, sino también de superar los roles y estereotipos que subyugan a las mujeres y, una vez más, imposibilitan su autodeterminación. 
El objetivo final es plasmar las legislaciones existentes del aborto en diferentes países y si éstas reconocen los derechos sexuales y reproductivos de las mujeres, como sujetos autónomos o, por el contrario, aún queda mucho para la igualdad real y efectiva entre hombres y mujeres - como sujetos portadores de derechos-, para lo que trataré de mostrar los compromisos internacionales en defensa de los derechos de la mujer y la falta de legislación que los secunde.

\section{Los argumentos sobre la interrupción voluntaria del embarazo}

Habitualmente, el debate sobre el aborto en la sociedad se ha simplificado en dos grandes posiciones: los grupos "pro vida" y los grupos "pro elección”. Desde ésta óptica, la incapacidad de resolver la cuestión de una regulación jurídica del mismo es más que evidente por la imposibilidad de encontrar un equilibrio entre los valores de ambos grupos.

Una reducción tan drástica, obvia argumentos que condicionan las legislaciones y enriquecen el debate. Las posturas sobre la legalidad o ilegalidad del aborto más destacadas cabe centrarlas en los siguientes tres argumentos.

La primera, defendida por el grupo antiabortista, se fundamenta en la hipótesis del valor inviolable de la vida del feto equiparándolo al de cualquier persona, que como tal, merece ser protegida. En pro de salvar la vida "del/de la niño/a inocente no nacido", la regulación legislativa propuesta, desde ésta perspectiva, es la prohibición absoluta del aborto.

Considerar el feto/embrión desde el momento de la concepción un ser humano se sustenta en argumentaciones morales que llevan a una serie de contradicciones. Es decir, con la intención de preservar esa vida (la del no nacido) cualquier supuesto sería inaceptable incluso en situaciones que vayan en prejuicio o puedan suponer la muerte de otra vida: la de la mujer que está gestando. Es el caso de violaciones sexuales, peligro de la vida de la madre o malformación del feto. El valor inviolable de la vida del no 
nacido obliga a prohibir radicalmente la interrupción voluntaria de la gestación, aunque sea a costa de la mujer que lo está gestando, eliminando así, el también valor inviolable de la vida de ella.

Una opción para no ser tan restrictivos es determinar cuándo el no nacido es un es un nascistorius ${ }^{1}$ o una persona, permitiendo determinar cuándo es un delito de homicidio el aborto, es decir, cuándo se atenta contra la vida de un ser humano.

Históricamente han existido múltiples intentos para definir el inicio del ser humano. Con Santo Tomás, por ejemplo, se introduce en Europa el hilomorfismo ${ }^{2}$ obligando a concretar el momento en que se produce la animación del feto, porque solo la muerte de un feto formado tenía alma y se consideraba homicidio. Ésta teoría de la animación retardada, que apareció en los Decretales de Gregorio IX, en 1234, delimitaba el delito a partir de los 45 y los 60 días. Si éste iba a ser niño el alma entraba a los 40 días y si, por el contrario, iba a ser niña, entraba a los 80 días (Sau, 1981).

Otro intento es la teoría de la preformación que se gesta desde finales del siglo XVII y mantiene la hipótesis que desde el momento de la concepción, con la primera célula, todo el contenido del futuro ser ya existe y lo único que ocurre con el período de gestación es que se expande y aumenta (Sau, 1981).

Ahora bien, como estas propuestas no están avaladas por una argumentación con fundamentación empírica científica que garantice la universalidad y validez de los mismos, es imposible justificar ésta postura desde una óptica objetiva. La imposibilidad de determinar científicamente, y no meramente conceptual, el momento concreto en que se origina una vida humana, no permite delimitar cuándo se ha realizado un homicidio, dejando la fundamentación de esta postura en consideraciones exclusivamente morales.

La segunda postura en el debate sobre la legitimidad o ilegitimidad del aborto voluntario considera la interrupción de la gestación como estado de necesidad, es decir, admitiéndolo en situaciones límites (García Pascual, 2007).

\footnotetext{
${ }^{1}$ Etapa previa al desarrollo del ser humano.

${ }^{2}$ Teoría de origen aristotélico que afirma la imposibilidad de la existencia del alma sin el cuerpo.
} 
Aunque desde esta óptica, el feto es un principio de vida humana y como tal debe ser protegido por el derecho, se acepta interrumpir el embarazo en caso de violación (por ser una experiencia traumática que produce trastornos psíquicos en la mujer), en caso de riesgo de malformación del feto o peligro para integridad de la madre.

Desde esta perspectiva se buscan mecanismos para proteger la vida del no nacido otorgándole unos derechos previos a su existencia y obviando los de la mujer. Subyace, detrás de ésta argumentación, un discurso "pro vida", al cual solo se le añaden limitaciones.

La tercera postura del debate se fundamenta en el derecho de la mujer a la autonomía reproductiva. La prohibición del aborto libre se concibe como una imposición: la obligación de ser madre.

Entendiendo la especial relación existente entre el feto y la madre cabe reconocer la necesidad de un equilibrio físico-psíquico durante el período de gestación (situación que claramente se reconoce en la segunda postura cuando se acepta el aborto en caso de violación).

La imposición de la maternidad supone una obligación y, desear interrumpir el embarazo, es actuar en contra de los propios deseos y voluntades, conllevando la limitación de la libertad de las mujeres y su reificación ${ }^{3}$. El derecho de la salud sexual y reproductiva de las mujeres queda en manos de terceros y no de ellas mismas, convirtiéndose en medios para la reproducción y perpetuación de la especie y no en seres humanos libres. El quebrantamiento de la autodeterminación femenina respecto a la procreación niega la plenitud moral del sujeto femenino como ser "responsable" capaz de cargar con las consecuencias de las propias elecciones - sea la de ser madre o la de elegir no serlo (Pitch, 2003).

\footnotetext{
${ }^{3}$ La cosificación de las mujeres es el resultado de una consecuencia lógica del discurso que le da un valor añadido a su cuerpo por la capacidad reproductiva, obviando otros factores que constituyen el propio sujeto-mujer.
} 


\section{Los diferentes niveles del debate}

Una legislación sobre el aborto consecuente con la emancipación de la mujer exige desvelar los diferentes niveles del debate para esclarecer la validez de éstos a la hora de justificar la prohibición del aborto o permitirlo.

Por un lado, en los dos primeros discursos - "pro vida" y "estado de necesidad"- se considera al sujeto con capacidad reproductora un medio para obtener un fin: dar la vida, mientras que en el tercero, la mujer es un fin en sí misma. En el primer caso, es identificada únicamente con su sexo, entendiéndola como una "máquina reproductora" que garantiza la perpetuación de la especie. En este caso, aquello importante es la reproducción per se y todo estaría orientado alrededor del proceso biológico. En cambio, plantear la interrupción voluntaria de la gestación como un derecho apoyado en la idea de autonomía de las mujeres supone considerarlas un fin en ellas mismas, sujetos libres y capaces de ser responsables de sus actos.

Este primer nivel del debate estaría sustentado en las concepciones de "la mujer como medio" y "la mujer como fin".

Por otro lado, existe otra diferenciación de niveles discursivos subyacente: la argumentación de los grupos antiabortistas le otorgan al feto el mismo estatus que a la madre, a quien le anulan la condición de sujeto, por tanto, de ciudadana. De esta manera, tanto el feto como la madre son considerados entes propios que llegados a la situación de un aborto voluntario, prevalece la vida del no nacido por encima del de la madre (por un principio de protección de la vida), aunque el primero aún no sea un ser humano vivo.

En cambio, en el derecho libre a interrumpir voluntariamente la gestación sí que se le reconoce a la mujer el estatus de sujeto autónomo ${ }^{4}$.

\footnotetext{
${ }^{4}$ Hay que destacar que cuando se admite la interrupción voluntaria por una cuestión de salud, prevalece el principio de protección de la vida, no concibiendo claramente a las mujeres como sujetos plenos a los que es necesario reconocer sus derechos (una de las excepciones es por riesgo de malformaciones del feto). Sin embargo, como se encuentren y cuál es su voluntad, de alguna manera sí que está presente.
} 
La postura que admite el aborto por cuestión de salud se encuentra en un punto intermedio.

Este nivel está centrado en a quién se le reconocen los derechos: si a las mujeres (dándoles libertad para escoger) o a los fetos (entendiéndolos como seres inocentes con derecho a la vida).

Es importante proteger la vida, pero no solo la del no nacido, también la de la madre que no quiere serlo y esto hace referencia, también, a la calidad de vida, a poder decidir cómo se quiere vivir sin tener la obligación impuesta a la maternidad por el mero hecho de estar embarazada.

Considerando que el debate más importante es el que se rige por el reconocimiento de derechos, hay que destacar que las leyes se establecen dentro de un concepto de ciudadanía, donde se fijan los derechos y deberes de los/as ciudadanos/as que forman parte del consenso social. Quedaría inevitablemente descartado el primer argumento "pro vida", ya que resulta imposible reconocer el derecho de ciudadanía a los fetos.

La legalización del aborto estaría en manos de la siguiente cuestión: ¿es un derecho político que atañe a la ciudadanía o es un derecho sanitario?

El eje del debate reside en si la mujer es o no un sujeto moralmente pleno, ciudadana que, como parte activa del contrato social, decide ejercer libremente un derecho y ser propietaria del propio cuerpo o, por el contrario, aquello que ha de prevalecer es la capacidad de procrear y solo en caso de una cuestión de salud, está justificado la intervención del periodo de gestación. 


\section{La construcción de identidad de género que subyace en el debate sobre el aborto}

Es importante analizar el concepto de "maternidad" que subyace, así como la construcción de la identidad de género que rigen las diferentes posturas delante de la legalidad o ilegalidad de la interrupción voluntaria del embarazo para entender porqué las legislaciones en el tema del aborto son más restrictivas que permisivas.

La protección de la vida humana se sustenta en el rol que se le asigna a la mujer en relación con la maternidad, dándole un valor añadido a la capacidad de procrear y, al mismo tiempo, privándole a ella de la autonomía como sujeto. Así, ser madre se convierte en aquello que da sentido a la vida de las mujeres $\mathrm{y}$, decidir no serlo supone "ir en contra de la propia naturaleza".

Por ende, el proceso biológico de la gestación y el parto no es concebido como una experiencia concreta y personal posible de las mujeres, sino que condiciona la construcción del género femenino, de todo el colectivo. La relación especial establecida entre el feto/embrión y la madre de manera biológica permite justificar toda una serie de connotaciones culturales atribuidas a las mujeres. De esta manera se reformula toda su identidad a partir de su sexo u órgano reproductor, reificándolas hasta el punto de ser concebidas como autómatas de la reproducción.

La maternidad sirve de mecanismo para ligar a las mujeres a una experiencia vital, que de posible se convierte en imprescindible, a la hora de concebirse como seres “completos". De no ser así son tachadas de "anti-naturales" por no cumplir con el considerado fin último de su existencia.

Esta concepción determinista de las mujeres sustenta múltiples roles y estereotipos, como es el rol de cuidadoras (encargadas de atender a los/as hijos/as), y de habilidades que per natura poseen única y exclusivamente: sensibles, emotivas, delicadas,... capaces de entender aspectos del mundo y de la naturaleza que vienen dados gracias a ser seres "elegidos" para el hecho biológico del embarazo y el parto.

No solo estos valores han sido extrapolados de una característica biológica que ha permitido destituir a las mujeres de todo poder de autodeterminación, sino que 
gracias a ello, se las ha recluido en la esfera doméstica, dejándolas sin capacidad en la toma de decisiones sociales tan importantes como el derecho al aborto, que las afectan directamente.

Garantizando la invisibilización social de las mujeres, la maternidad ha sido la forma de simplificar su existencia -hay un valor añadido al proceso biológico, pero no a ellas mismas por ser las co-protagonistas de éste- y, al mismo tiempo, de dotarla de una concepción peyorativa.

Ejemplo de esto son los simbolismos de género utilizados en la ciencia que muestran la perversión cultural en un ámbito de conocimiento considerado objetivo, pero que sustentado en dicotomías de naturaleza/cultura ${ }^{5}$, ha aportado recursos para el progreso de dominación masculina.

Concretando, en la concepción orgánica de la naturaleza en el Renacimiento, el Sol se asociaba a la masculinidad y la Tierra a la feminidad, siendo ésta última "una madre cariñosa" y al mismo tiempo "salvaje e incontrolable". (Harding, 1996:101)

El sexo define al género femenino de manera asimétrica, permitiendo desvalorizar y jerarquizar a las mujeres mediante el proceso biológico de la maternidad y los argumentos científicos enraizados en un sistema patriarcal que subordina las mujeres a los hombres.

Siendo esto así resulta más que fácil atribuir a las mujeres capacidades innatas para la educación y el cuidado al mismo tiempo que se les asigna un destino ya marcado: el de ser madres. Hechos que quedan enlazados a vivencias vitales como son la maternidad y también a una conciliación "exclusiva de las mujeres" de la vida personal, familiar y laboral con jornadas parciales, contratos laborales precarios, salarios más bajos y dificultades para la promoción profesional.

Así pues, tanto dar la vida como protegerla, desde esta perspectiva, elimina toda posibilidad de autodeterminación femenina, porque la construcción de su identidad "viene dada" y sólo, planteando una igualdad efectiva es viable romperla, ya que es la

\footnotetext{
${ }^{5}$ La naturaleza es símbolo de la feminidad mientras que la cultura, campo de conocimiento racional, es enlazado con los hombres.
} 
única manera de concebirla como sujetos autónomos y responsables plenamente morales capaces de elegir igual que los hombres.

El derecho a abortar voluntariamente pretende dar una significación social a la maternidad, dejando de ser tratado como solo un fenómeno biológico, desapareciendo la maternidad en esclavitud (Sau, 1981).

Pero, sin embargo, tanto la postura antiabortista como la de aceptación del aborto por una cuestión de necesidad, identifican a la mujer con la madre, y dificultan la existencia de una legislación sobre la interrupción voluntaria del aborto que no vincule necesariamente a la mujer con la experiencia de la maternidad.

\section{Las legislaciones sobre la interrupción voluntaria del embarazo en el mundo}

Después de todo esto se puede comprender mejor el porqué de las diferentes políticas legislativas sobre el aborto existente en el mundo, debido a que la construcción cultural de la identidad de género fundamenta y condiciona una parte importante de las posturas sobre el derecho al aborto.

Generalmente, no se produce una regulación del derecho al aborto apoyado en la idea de autonomía de las mujeres, sino que más bien se tiende a su legalización para controlar un fenómeno clandestino o evitar "males mayores" por cuestiones de salud.

Ahora bien, existen avances producidos respecto a la libertad de las mujeres y el reconocimiento de sus derechos humanos, mostrando la necesidad de eliminar todo tipo de imposiciones en la construcción de su identidad, plasmándolo legislativamente. Muestra de ello es la aportación que, en el ámbito Internacional, hace la Declaración de Beijing (IV Conferencia Mundial sobre las mujeres, 1995), la cual marca un punto de inflexión en el posicionamiento de los diferentes países en el debate sobre el aborto. Los gobiernos de 189 países participantes estaban convencidos que "el reconocimiento explícito y la reafirmación del derecho de todas las mujeres a controlar todos los 
aspectos de su salud, en particular de su propia fecundidad, es básico para su empoderamiento" (Naciones Unidas; 1995: 43).

En esta Declaración, la salud reproductiva se entiende como "un estado general de bienestar físico, mental y social, y no de mera ausencia de enfermedades o dolencias" y, en consecuencia, entraña "la capacidad de disfrutar de una vida sexual satisfactoria y sin riesgos y de procrear, y la libertad para decidir hacerlo o no hacerlo, cuándo y con qué frecuencia” (Naciones Unidas; 1995: 44).

Al mismo tiempo, también afirma que ésta definición de derechos reproductivos abarca ciertos derechos humanos que "se basan en el reconocimiento básico de todas las parejas e individuos a decidir libre y responsablemente el número de hijos, el espaciamiento de los nacimientos [...] y el derecho a alcanzar el nivel más elevado de salud sexual y reproductiva" sin por ello "sufrir discriminación, coacciones ni violencia" (Naciones Unidas; 1995: 45).

Desde un ámbito internacional como es la Plataforma de Acción de Beijing, la salud reproductiva se refiere no sólo a la salud sexual, sino también a la interrupción voluntaria de la gestación. Procrear, o no, se trata de una decisión libre y responsable exenta de coacciones y discriminaciones. La autodeterminación femenina es la clave para la construcción de un mundo justo e igualitario.

Estaríamos de acuerdo con Dworkin al reconocer el derecho y la responsabilidad de las personas a enfrentarse de manera autónoma a los asuntos fundamentales que atañen a sus propias vidas, respondiendo a sus convicciones y conciencias.

El derecho a tener control sobre las cuestiones relativas a su salud sexual y reproductiva y el de decidir libremente respecto a todo lo que atañe a esas cuestiones son considerados, por tanto, derechos humanos de la mujer, No solo se plantea desde una óptica dónde la mujer es un ser responsable y consecuente de sus acciones, sino que además se le reconoce la capacidad de disfrutar de una vida sexual plena y satisfactoria.

Ahora bien, la realidad contrasta con los planteamientos teóricos si observamos los textos del Derecho Internacional, debido a que en ninguno se plasma el derecho al 
aborto, sino más bien lo contrario, se reconoce el derecho a la vida, mostrándose que la principal razón es salvar la vida del no nacido, entendida como una vida humana digna de protección o por la permisividad por razones sanitarias.

Unas legislaciones coherentes y acordes a los compromisos internacionales, tendrían que regirse por el reconocimiento de la autonomía de las mujeres, y no por normativas jurídicas restrictivas que otorgan el derecho al aborto con limitaciones arbitrarias para no ir en contra de los discursos moralistas de protección de la vida humana del feto.

Y esto es mucho más necesario cuando añadimos toda esta serie de datos importantes que condicionan la existencia de estas regulaciones al mismo tiempo que exigen un replanteamiento asentado en otros axiomas que la "protección de la vida". ¿Qué vida hay que proteger la del no nacido -aún no ser humano- o la de la mujer que lo ha engendrado?

Como plasman las diferentes legislaciones prevalece en muchos casos la vida del feto/embrión sobre la vida de la madre ${ }^{6}$.

Por un lado, el índice por mortalidad materna según la Organización Mundial de la Salud es de unas 529.000 mujeres al año, sin contar que cada vez que una mujer muere al dar a luz, otras 20 sufren lesiones, infecciones o enfermedades (unos 10 millones de mujeres cada año).

Cinco complicaciones directamente relacionadas son responsables de más del $70 \%$ de las muertes maternas: hemorragias (25\%), infecciones (15\%), abortos peligrosos (13\%), eclampsia (hipertensión arterial producida que provoca convulsiones $-12 \%)$ y parto obstruido $(8 \%)$.

Por otro lado, según el Informe sobre la salud en el mundo del año $2005^{7}$ de la OMS de una estimación "de 123 millones de mujeres que logran quedarse embarazadas

\footnotetext{
${ }^{6}$ La expresión "en caso de peligro para la madre" queda limitada a una cuestión de riesgo de muerte, pero obvia los posibles trastornos mentales de "estar obligada" a continuar con un embarazo no deseado.
} 
al año, 87 millones se producen de manera involuntaria" y "de los 211 millones de embarazos producidos al año, 46 millones acaban en abortos provocados" (OMS, 2005: $55)$.

Figura 1

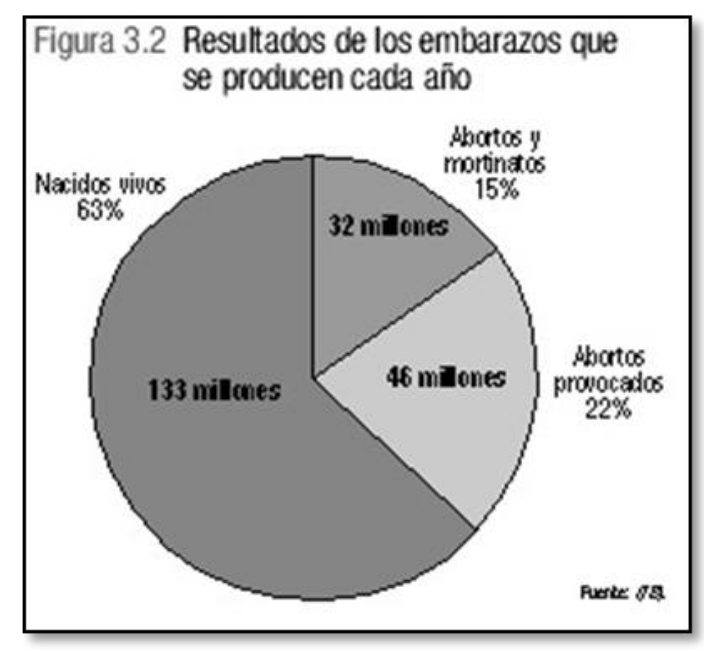

Fuente: Organización Mundial de la Salud

Además, "las mujeres que han sido víctimas de agresiones sexuales [las cuales tienen menos probabilidades de estar en condiciones de utilizar métodos anticonceptivos a menudo temen haberse quedado embarazadas y posponen el momento de someterse a exámenes médicos o de solicitar atención sanitaria” (Op. cit.: 53).

Otro dato importante, al hilo del anterior, es el gran número de agresiones sexuales a menores y mujeres, especialmente en algunos países con conflictos armados y extrema pobreza, entre otros factores. Todo ello dificulta su detección o denuncia y no son fácilmente estimables.

${ }^{7}$ OMS, Informe sobre la salud en el mundo (2005). En el informe más reciente (2008) no existen datos estadísticos que puedan reemplazar a estos. 
La existencia de un alto porcentaje de abortos clandestinos ${ }^{8}$ y el uso de técnicas abortivas de riesgo como son la inducción de sustancias que provocan contracciones uterinas -agua jabonosa, desinfectante casero, perejil,...-preparaciones orales, aborto por masaje -presionando el vientre de la embarazada- son prácticas desesperadas de mujeres que viven en lugares donde el derecho al aborto no es reconocido o les resulta imposible acceder por sus circunstancias socioeconómicas, optando por arriesgar su propia vida aunque les cueste la muerte.

Así pues, analizando las regulaciones legislativas en materia de interrupción voluntaria de la gestación, el $61 \%$ de la población mundial vive en países donde el aborto inducido está permitido aunque sea con limitaciones y sólo el $26 \%$ reside en lugares donde está esencialmente prohibido (Center for Reproductive Rights, 2007).

Observando el mapa anterior y mediante una clasificación general alrededor de las leyes sobre el aborto en el mundo del Center for Reproductive Rights, la interrupción voluntaria del embarazo está regulada de acuerdo a los factores siguientes:

Algunas legislaciones lo permiten cuando se trata de evitar un grave peligro para la vida o salud física o psíquica de la embarazada. En tales casos no vale la palabra ni de la paciente ni del/de la médico/a que practica la interrupción de la gestación, para poder abortar es necesario un dictamen emitido por un/a especialista distinto. Por tanto, no se contempla a la mujer como un sujeto moralmente pleno autónomo y responsable, es decir, no se la concibe como a una persona capaz de responsabilizarse de sus acciones, siendo necesario alguien que la tutele y decida por ella.

Otro caso son los países que "permiten explícitamente el aborto para proteger la salud mental de la mujer, además de su vida y su salud física.” La interpretación del término "salud mental" es variable, pudiéndose referir a los trastornos producidos por una acción considerada delito como es una violación o al estado de tensión provocado por unas circunstancias socioeconómicas difíciles (Center for Reproductive Rights, 2007).

\footnotetext{
${ }^{8}$ Muchos países han publicado estimaciones de abortos clandestinos, pero en realidad es imposible cuantificarlos aunque se reconoce el alto porcentaje en países en vías de desarrollo.
} 
En cambio, hay legislaciones que permiten el aborto por razones socioeconómicas, atendiendo a factores como recursos económicos, situación personal, número de hijos/as. Detrás de estas regulaciones legales suelen existir planteamientos de control de la natalidad en base a políticas socioeconómicas y no argumentos en pro de la libertad de decisión de la mujer.

Las leyes más permisivas son las de países que no existe restricción alguna para permitir el aborto, pero fijan unos plazos. El problema fundamental de las leyes de plazos para la interrupción voluntaria de la gestación es la restricción de éstos, no hemos de olvidar que en un primer momento se puede desconocer el hecho de estar embarazada y, además, al ser una situación sobrevenida, la mujer posiblemente esté desorientada, tardando en reaccionar.

También hay legislaciones que reconocen explícitamente el derecho al aborto en caso de posible malformación del feto, tanto física como psíquica, pero se exige informe de especialista distinto del que atiende a la mujer o practica la interrupción del embarazo.

Después de un análisis general de las legislaciones mundiales sobre el aborto, es importante destacar algunas de ellas.

Una de las más importantes por su planteamiento "pro vida" es la de México. Ya son 18 estados que han hecho reformas constitucionales para proteger la vida desde la fecundación. De todos ellos, es destacable la aportación de Chiapas, que con la Ley General de Maternidad y Paternidad Responsable, reconoce la vida desde la concepción y substituye la pena de cárcel por un tratamiento médico integral (psicológico) que "será proporcionado por las instituciones de salud a fin de ayudarles a superar los efectos de la intervención y reafirmar los valores humanos de la maternidad", además de cumplir la pena haciendo trabajos comunitarios.

Posiblemente este sea, uno de los ejemplos más radicales y, curiosamente, de los más actuales.

Otro país, como es Irlanda, proclama la personalidad del feto en su texto constitucional y prohíbe el aborto, pero se han aprobado cambios constitucionales 
permitiendo a sus ciudadanas interrumpir voluntariamente la gestación en el extranjero (García Pascual, 2007).

También hay que destacar, por su actual modificación, el caso de la legislación sobre el aborto en España que establece una ley de plazos, permitiendo el aborto libre hasta las 14 semanas, informando previamente sobre la interrupción del embarazo, los derechos de la mujer así como las prestaciones y ayudas públicas a la maternidad, con un periodo de reflexión de al menos tres días ${ }^{9}$.

Se permite la interrupción hasta las 22 semanas en caso de grave riesgo para la vida o salud de la mujer o malformaciones del feto. A partir de éste periodo, sólo se podrá interrumpir el embarazo sin limitaciones si hay malformación incompatible con la vida del feto o padece alguna enfermedad grave e incurable, pero con un diagnóstico médico previo contrastado (dos especialistas o un comité clínico formado por tres médicos).

En menores de entre 16 y 17 años la decisión será de éstas pero tendrán que informar a uno de los representantes legales. Si alega fundadamente que esto puede suponer situaciones de conflicto grave se prescindirá de esta información.

Por tanto, para concluir es imprescindible reconocer los avances, inconcebibles hasta hace unos años, en el reconocimiento de los derechos de la mujer de algunas legislaciones, pero siguen siendo insuficientes después de mostrar la situación de éstas en lo referente a sus derechos sexuales y reproductivos en el panorama internacional.

La equidad entre mujeres y hombres implica necesariamente el reconocimiento de las primeras como sujetos moralmente plenos, autónomos y responsables de sus actos, siendo necesario denunciar y rechazar todo tipo de legislaciones privativas de los derechos de las mujeres, que además, contradicen los compromisos internacionales.

Quizás el reconocimiento del derecho al aborto por una "cuestión de necesidad" significa un paso, pero sólo por el hecho de reconocer un derecho, ya que en

\footnotetext{
${ }^{9}$ Se sigue dando un periodo de reflexión como si la mujer optara directamente por abortar sin haber hecho una reflexión antes. Al mismo tiempo, queda en el aire la objeción de conciencia de los/las médicos.
} 
ésta situación sigue quedando camino hacia la libertad de las mujeres, hacia su autonomía moral plena, porque siguen sin poder elegir y decidir sobre su propio cuerpo y su futuro.

Es evidente que detrás de los lentos pasos de las legislaciones en materia de interrupción voluntaria del embarazo sigue pesando con mucha fuerza todos los roles que han sido asignados históricamente a las mujeres, construyendo su identidad ligada inevitablemente con la maternidad. Por ello, se puede entrever si contraponemos los acuerdos internacionales en dicha materia y las legislaciones de diferentes países ciertas contradicciones. 


\section{BIBLIOGRAFÍA}

- Borrell, F. y Arisa, J. R. (Comitè Consultiu Bioètica de Catalunya, 2009): Informe sobre la interrupción de la gestación. Barcelona: Generalitat de Catalunya, Departament de Salut.

- Center For Reproductive Rights (2007): "Leyes sobre el aborto en el mundo" (briefing paper), [en línea] Disponible en: http://158.109.129.18/centreantigona/docs/articulos/Lleis\%20sobre\%20l'abort\%20al\%2 Omón.pdf [19/02/2010].

- Dworkin, R. (1998): El dominio de la vida: una discusión acerca del aborto, la eutanasia y la libertad individual. Barcelona: Ariel, S.A. Editorial.

- García Pascual, C. (2007): “Cuestiones de vida o muerte. Los dilemas éticos del aborto". En Derechos y libertades, no. 16, pp.181-209.

- González, C. (2009): "Sexualidad y aborto, ¿cuestión de salud?, ¿cuestión de derechos?”. En Anuario de Derechos Humanos. Nueva Época, no. 10, pp.285-329.

- Harding, S. (1996): Ciencia y feminismo. Madrid: Ediciones Morata, S.L.

- Ministerio de Igualdad (2010): "El Senado aprueba la Ley de Salud Sexual y Reproductiva e Interrupción voluntaria del embarazo", [en línea] Disponible en: http://www.migualdad.es/ss/Satellite?c=Page \&cid=1193047406588\&language=cas_ES\&np =3\&pagename=MinisterioIgualdad\%2FPage\%2FMIGU_listadoNotasPrensa [25/02/2010]. - Naciones Unidas (1995): "Informe de la Cuarta Conferencia Mundial sobre la Mujer, Beijing [en línea] Disponible en: http://www.uneca.org/daweca/Documents/Beijing\%20Declaration\%20\&\%20Platform\% 20for\%20Action\%20SPANISH.pdf [28/04/2010].

- Organización Mundial de la Salud (2005): "Informe sobre la salud en el mundo", [en línea] Disponible en: http://www.who.int/whr/2005/es/ [24/02/2010].

- Pitch, T. (2003): Un derecho para dos. La construcción jurídica de género, sexo y sexualidad. Madrid: Editorial Trotta.

- Sau, V. (1981): Diccionario ideológico feminista. Barcelona: Icaria Editorial.

- Chiapas (2009): Ley General de Protección a la Maternidad y a la Paternidad Responsable. 